\title{
AOIR
}

Selected Papers of \#AoIR2020:

The $21^{\text {st }}$ Annual Conference of the

Association of Internet Researchers

Virtual Event / 27-31 October 2020

\section{SOWING SEEDS OF DISTRUST: VIDEO GAME PLAYER PERCEPTIONS OF COMPANIES IN ONLINE FORUMS}

\author{
Christine Tomlinson \\ University of California, Irvine \\ Maria J. Anderson-Coto \\ University of California, Irvine
}

\section{Extended Abstract}

As an increasingly popular form of entertainment, video games offer a variety of options for players, from major AAA titles from big development studios to indie games created and released by small teams. Many larger companies promote the growth of fandoms through specific platforms and major game titles that tend to get people excited. Fans in turn start acquiring an identity as part of their self-expression (Claudiu \& Andreea, 2014) and begin determining their relationship with the company. Within this landscape, what does fan commitment look like? More specifically, how do fans negotiate trust around these companies? Why might fans lose confidence in a company?

Based on our findings, there has been a growing distrust in the ability of video game companies to produce enjoyable products without taking advantage of the user. Consumers have become increasingly critical, leading to lowered expectations of quality, discouraging people from preordering, and reaffirming a perceived lack of value. This does not stop with general gaming audiences, extending to the experiences and interpretations of esports fans.

Online communities have been widely studied in both games (Steinkuehler \& Williams, 2006) and business. Virtual communities allow businesses to get to know their customers, build relationships, and increase consumer loyalty (Hagel 1999; Kardaras et al, 2002). Video game companies have recognized these communities as important spaces for spreading the word for their games and maintaining bonds with their audience (Ruggles, Wadley, \& Gibbs, 2005). While consumers can "love" a brand, this is largely influenced and entangled with the perception of what that company can do for them (Batra, Ahuvia, \& Bagozzi, 2012). Trust and attachment, however, can work as separate experiences,

Suggested Citation (APA): Tomlinson, C., \& Anderson-Coto, M.J. (2020, October 28-31). Sowing seeds of distrust: Video game player perceptions of companies in online forums. Paper presented at AolR 2020: The 20th Annual Conference of the Association of Internet Researchers. Virtual Event: AolR. Retrieved from http://spir.aoir.org. 
with younger consumers feeling attachment toward a brand, but not necessarily trusting them (Bidmon, 2017).

This study is the product of two other projects, one looking at video game player behavior in 2016 and one looking at esports fans in 2019. Both investigated fan behaviors and online conversations around issues of identity, experience, and interest. In both cases, Reddit was the site of analysis to understand how video game players and esports fans talk about their interests and experiences, ultimately building cultures. The initial 2016 study included approximately 2,000 online posts and the 2019 study included 500 posts. While neither project specifically set out to discover how online forum users assess video game companies, this theme emerged through coding patterns.

From our analysis, many players discuss video game companies as beacons of obsession with profit, leaving audiences feeling forgotten or seen as a means to a financial end. While financial considerations are often at the root of consumer concerns, distrust appears in a few specific ways: predicting quality and content, discouraging preorders, reaffirming lack of value, and criticizing player support services. When it comes to new game announcements, consumers are often poised not only to experience hype, but to counter hype with what appear to be attempts at grounding the rest of the audience. For example, consumers have noted a trend in trailer reveals that look much more polished than the final product. This has, in turn, led to harsh predictions of game quality on the part of many viewers, with expectations that games will not live up to the hype being generated by the audience.

This reluctance to trust companies extends to esports fans as well. Because developers are also heavily involved in the esports scene, the audience - often in dual roles as both viewers and players of the games - gains a wide breadth for criticism. In this space, developers and organizers alike are seen as trying to economically exploit their audience. Thus, their critique is not only directed at the developer as a game-maker, but also as the entity responsible for the game's esports scene. Consumers call into question decisions made for esports teams, interactions between game developers and esports organizers, and updates to the game that alter the gameplay. Fans frequently attribute these decisions to a desire for profit and a lack of concern for the audience.

This leads directly into general reactions to and policing of specific fan behavior. In particular, video game fans are cautious about encouraging or supporting preorder habits. In discussions about upcoming games, especially where collector's editions are involved, commenters disagree about appropriate approaches to new releases. While many fans maintain a general enthusiasm to get games upon release, preordering has become a ground for argument and discord. Preordering is increasingly viewed as rewarding companies for their bad behavior. In these cases, consumers point to game companies' tendencies to present false game footage, lie about features, and to seemingly exploit fans by increasing microtransactions or requiring payment to unlock DLC that already exists in the game's code. In esports, fan behavior is affected by both the games and by esports events. While the audience is excited for events and leagues, they resent the broadening of esports for casual, sometimes even non-player viewership. This also tends to be labeled as another method for developers to make more money at the expense of the "true" fanbase. 
For many forum users, negative news about games or esports events reinforces the distrust and caution being taken. This is not just true of the games that they anticipated would not meet their expectations, but also for game and esports companies overall. In some ways, this becomes integrated into the broader culture for video game consumers and esports fans. The idea that certain companies will include more features that incentivize players to spend money, specific developers will release games with bugs, or that esports events will probably have technical issues is regarded as common knowledge. Many actions and decisions made by companies, then, become associated with carelessness.

While these sentiments are not expressed by all consumers, players, and viewers, they are increasingly present in the overall discourse of video game and esports audiences. This should be of concern for companies, since consumers' feelings and expressions in public spaces such as forums might potentially form and promote negative associations toward them and specific games or franchises.

\section{References}

Batra, R., Ahuvia, A., \& Bagozzi, R. P. (2012). Brand love. Journal of Marketing, 76(2), 1-16. https://doi.org/10.1509/jm.09.0339

Bidmon, S. (2017). How does attachment style influence the brand attachment - brand trust and brand loyalty chain in adolescents? International Journal of Advertising, 36(1), 164-189.

Claudiu, M., Andreea, P. (2014). Brand as a mean of consumer self-expression and desired personal lifestyle. Procedia- Social and behavioral sciences, 108, 103107.

Hagel, J. (1999). Net gain: Expanding markets through virtual communities. Journal of Interactive Marketing, 13(1), 55-65. https://doi.org/10.1002/(SICI)15206653(199924)13:1<55::AID-DIR5>3.0.CO;2-C

Kardaras, D., Karakostas, B., \& Papathanassiou, E. (2003). The potential of virtual communities in the insurance industry in the UK and Greece. International Journal of Information Management, 23(1), 41-53. https://doi.org/10.1016/S0268-4012(02)00067-1

Ruggles, C., Wadley, G., \& Gibbs, M. R. (2005). Online community building techniques used by video game developers. Proceedings of ICEC 2005.

Steinkuehler, C. A., \& Williams D. (2006). Where everybody knows your (screen) name: Online games as "third places." Journal of Computer-Mediated Communication, 11, 885-909. 